\title{
Metal evaporated tape: state of the art and prospects
}

\author{
S.B. Luitjens ${ }^{\text {a. }{ }^{*}, \text { S.E. Stupp }}{ }^{a}$, J.C. Lodder ${ }^{b}$ \\ a Philips Research Laboratories, Professor Holstlaan 4, 5656 AA Eindhoven, The Netherlands \\ ${ }^{h}$ MESA Research Institute. Unicersing of Twente. P.O. Box 217, 7500 AE Enschede, The Netherlands
}

\begin{abstract}
Thin metallic films are the first choice for media in advanced rigid disk systems. For helical scan tape recorders thin metal films have become more important. The high signal-to-noise ratio per unit of track width allows very high densities. The preparation techniques and materials properties of the thin-film coating of metal evaporated (ME) are described. Important aspects are the size of the grains, the geometry of the columnar structure and the associated anisotropy. The consequences for the recording process are explained. Tribological and corrosion properties also help to determine the usefulness of a recording tape. Corrosion, wear, protection layers and lubricating organic films are briefly discussed.
\end{abstract}

\section{Introduction}

In the last few decades the storage density of all magnetic recording systems have been increasing continuously. A density in excess of $1 \mathrm{bit} / \mu \mathrm{m}^{2}$ is expected in commercial rigid disk systems using thin metallic films as a storage layer and a thin-film MR head. In tape storage systems (for video and data) both particulate coatings and thin metallic films are used as the storage layer. This paper discusses the development of metal evaporated (ME) tape; for further information on this topic see Refs. [1-4].

The first generation of commercial ME tapes was introduced in 1989 for the Hi-8 video system [1,2]. This was followed by the Scoopman digital recorder with a stampsized cassette [5]. In 1993 a new digital video system was proposed (Digital Video Cassette, DVC) [6] which uses an improved ME tape with a $4 \mathrm{~dB}$ higher S/N [4].

An important consideration in system design is the trade-off between the amount of data reduction, playing time, and the cassette size. For both the $\mathrm{Hi}-8$ and DVC systems equivalent metal particle (MP) tapes have been developed which meet these requirements. There is an ongoing competition between ME and MP tapes, and current MP tapes are cheaper than ME tapes. The Scoopman system illustrates the potential advantages of ME tapes. which may be important in future systems. The need for extremely small size combined with long playing times, required a tape with a very high signal-to-noise ratio with very thin magnetic and total tape thicknesses. A summary of some ME tape parameters is given in Table 1.
The key developments in the commercialization of ME tapes have been the control of the surface roughness, the development of proper lubricants, and controlled oxidation resulting in the appropriate composition. We begin our discussion with the materials properties.

\section{Materials, structure and anisotropy}

ME tapes have been prepared by reactive oblique evaporation of $\mathrm{Co}_{80} \mathrm{Ni}_{20}$ or $\mathrm{Co}$ with localized supply of oxygen. The morphology (shape and size of the banana-shaped columnar structures, presence of voids and or antiferromagnetic $\mathrm{CoO}$ ) and microstructure (crystal shape and size, orientation of the crystals) are important for the magnetic behaviour of the tape. Consequently the ME tapes show structural and magnetic features over a variety of length scales depending on the choice of materials and deposition parameters. Fig. 1 shows an SEM image of the side of a crack in a $\mathrm{COO} \mathrm{ME}$ tape [7], in which banana-shaped columns can be seen. The picture shows two Co-O layers and a diamond-like carbon (DLC) film on top for protection against wear and corrosion [7]. Magnetization, coercivity and anisotropy are strongly connected with the deposition parameters such as the deposition angle, rate, temperature and oxygen supply.

In the early ME tapes $\mathrm{Co}-\mathrm{Ni}-\mathrm{O}$ was the material used. The material is deposited using a continuously varying angle of vapour incidence which leads to the mesoscopic banana-shaped column morphology (Fig. 1). There are indications [9] that a column consists of aggregates of fibres $(<10 \mathrm{~nm})$ consisting of ferromagnetic and non-ferromagnetic structures (grains, clusters of grains, etc.). Between the fibres and the bundles of fibres there are open areas (voids) that reduce the magnetic volume of the tapes.

Corresponding author. 
Table 1

Some parameters of (commercial) ME tapes ( $H_{\mathrm{c}}$ and $M_{\mathrm{s}}$ are intrinsic values in the easy axis direction)

\begin{tabular}{llllllll}
\hline & $\begin{array}{l}\text { Thickness } \\
(\mu \mathrm{m})\end{array}$ & Substrate & $\begin{array}{l}\text { Width } \\
(\mathrm{mm})\end{array}$ & $\begin{array}{l}H_{\mathrm{c}} \\
(\mathrm{kA} / \mathrm{m})\end{array}$ & $\begin{array}{l}M_{\mathrm{s}} \\
(\mathrm{kA} / \mathrm{m})\end{array}$ & $\begin{array}{l}\text { Coating } \\
(\mathrm{nm})\end{array}$ & Material \\
\hline Hi-8 & 11 & PET & 8 & 90 & 420 & 200 & CoNiO \\
DVC & 7 & PET & 6.35 & 120 & 640 & 150 & CoO \\
Scoopman & 4.8 & Polyaramide & 2.5 & & & & \\
\hline
\end{tabular}

The authors have found crystalline grains of $3-5 \mathrm{~nm}$ [8]. These films seem not to be totally crystalline but partly amorphous, and several oxide and metallic phases have been observed.

The dependence of the saturation magnetization $M_{\mathrm{s}}$, coercivity $H_{\mathrm{c}}$, signal, and noise on the process parameters has been discussed in Ref. [2]. There is a direct relationship between $\mathrm{H}_{\mathrm{c}}$ and the $\mathrm{O}_{2}$ content (due to the increased decoupling of grains) and an inverse relationship between $M_{\mathrm{s}}$ and the $\mathrm{O}_{2}$ content (due to a reduction in the Co content and an increase in the $\mathrm{CoO}$ content). To study further the relation between the deposition angle, oxygen concentration, magnetic properties and recording performance, a mini-roll coater has been developed [9] and basic experiments have been performed. Auger depth profiling showed that the $\mathrm{Co} / \mathrm{Ni}$ ratio is constant over the total thickness of the film. The $\mathrm{O}_{2}$ concentration is enhanced at the top $(30 \mathrm{~nm})$ and the bottom $(10 \mathrm{~nm})$. The relation between the deposition rate, the magnetic properties and structural features has been studied. Three tapes with deposition rates of 21 (A), 14 (B) and $7(\mathrm{C}) \mathrm{nm} / \mathrm{s}$ have been prepared (note that these rates are an order of magnitude smaller than the deposition rates of commercial media). The magnetic easy axes were varied from $23^{\circ}(\mathrm{A})$ to $47^{\circ}$ (C) with respect to the film plane (these are intrinsic measurements which are corrected for sheet demagnetization [13]). The columnar tilt angle relative to the film plane becomes larger for the more strongly oxidized films (sample C). This shows how the random surface diffusion is more limited by the oxygen at low deposition rates leading to a columnar growth towards the film normal because of the conservation of momentum of the incoming atoms. From electron diffraction we concluded that $\mathrm{CoO}$ and $\mathrm{NiO}$

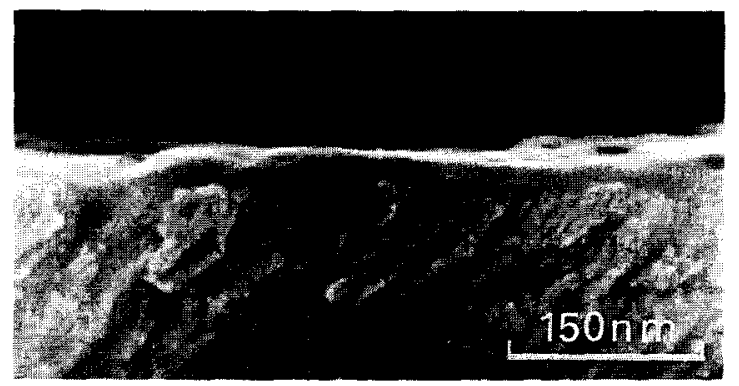

Fig. 1. SEM cross section of an ME tape [7]. phases are present in all tapes. (The oxidized state is important in determining the coupling between the fibres, columns and even within the fibres. This will be discussed later in the context of noise studies.) The crystal size also varies with the deposition rate and was $7 \mathrm{~nm}$ for the highest rate and $22 \mathrm{~nm}$ for the lowest [9]. As part of the magnetic characterization of the samples, VSM measurements have been performed at low temperatures $(40 \mathrm{~K})$. If the material is cooled down in a magnetic field through the Néel temperature of the antiferromagnetic Co-O $\left(T_{N}=293\right.$ $\mathrm{K})$ an interaction between this phase and the ferromagnetic Co phases arises. This is a unidirectional anisotropy [10] and is manifest in a shifted hysteresis loop [9]. Similar results have been found for commercial tapes [11].

Recent advanced ME tape is produced by oblique deposition of $\mathrm{Co}-\mathrm{O}$ layers [7]. Some experimental observations $[12,4]$ show that these films have a microstructure similar to the $\mathrm{Co}-\mathrm{Ni}-\mathrm{O}$ films: an hcp $\mathrm{Co}$ phase and $\mathrm{CoO}$ crystals of about $5 \mathrm{~nm}$ are found. The easy axis of magnetization is about $40^{\circ}$ from the tape plane. These tapes have shown sharp transitions at $100 \mathrm{kfrpi}$. The high recording densities are a result of the microstructure as well as the higher $M_{\mathrm{s}}$ values (due to the higher Co content) in these media.

The oblique columnar structure of ME tapes gives rise to very well oriented, highly uniaxial media. This results in extremely square hysteresis loops. The consequences of these properties on the recording process will be discussed in the next section. Fig. 2 shows the intrinsic hysteresis properties of a commercial $\mathrm{Co}-\mathrm{Ni}-\mathrm{O} \mathrm{ME}$ tape with the internal field in the easy axis direction $\left(35^{\circ}\right.$ from the tape plane). The intrinsic loop is obtained by correcting for the sheet demagnetization during the VSM measurement as described in Ref. [13]. The squareness is very close to 1 .

From a materials perspective there are several areas in which additional research is necessary to further advance our understanding and to help improve the properties of these media. From the studies of in-plane as well as perpendicular hard disk media it appears that microstructural features are important in determining the noise and recording densities. In $\mathrm{ME}$ tape this translates into a need to understand the influence of process parameters and substrate surface structures on the final thin-film structure. The oxidation process, which is critical in determining the macroscopic magnetic properties $\left(M_{\mathrm{s}}\right.$ and $\left.H_{\mathrm{c}}\right)$ as well as the intergranular coupling, is poorly understood. Further 


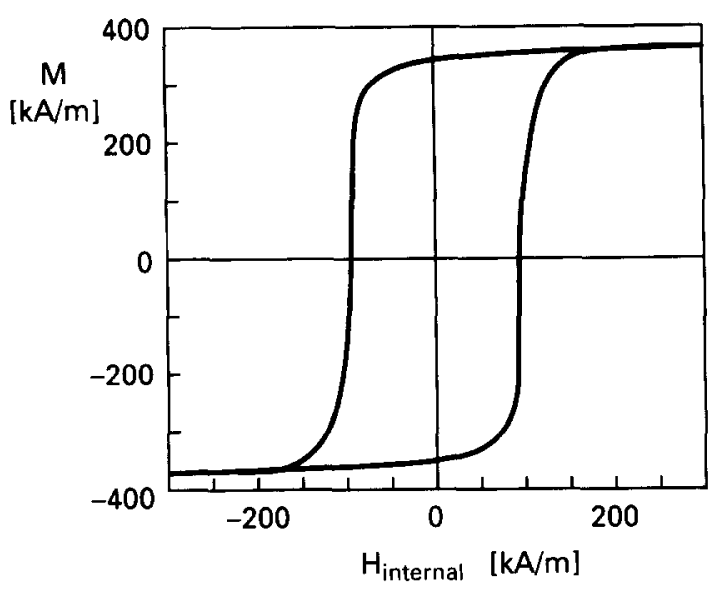

Fig. 2. Intrinsic hysteresis loop in the easy axis direction.

studies of the influence of the substrate and seed layers on the film morphology and structure are required. Finally, the role of non-magnetic regions such as voids, needs to be elucidated so that the effective packing fraction can be increased while maintaining the superior recording performance associated with ME tapes.

\section{Recording properties}

Experimentally, ME tapes have the highest output signals at short wavelengths of any flexible recording media [2]. In addition, ME tapes also exhibit a characteristic asymmetric pulse shape, and various asymmetries in the recording process (for example, in the output as a function of the direction of relative head-to-tape motion, or so-called good and bad recording directions) $[2,14]$. These properties can be understood in the context of the material properties discussed in Section 2. The extreme uniaxial anisotropy associated with the mesoscopic columnar structure in ME tapes gives rise to an oblique recording process which is close to one-dimensional (1D) in nature [14].

An oblique easy axis angle gives rise to asymmetric pulse shapes since the convolution of the head sensitivity function with the medium magnetization in the read process includes both $x$ - and $y$-components of the head field. Similarly, the good and bad recording directions can be understood by considering the projection of the head fields along the oblique medium easy axis in the write process. If writing is assumed to occur at the trailing edge of the head where $H_{\text {total }}=H_{c}$, in the good direction of head-to-tape motion, the magnitude of the projected total field gradient is larger, leading to sharper transitions (and thus larger output signals at shorter wavelengths) than the bad direction.

The effect of recording in the good and bad directions on the recorded bits can be studied with MFM observations. Fig. 3 shows written bits for recording in the two directions. The same current is used for writing the bits.
The distance between transitions ( = bit length) is about $0.33 \mu \mathrm{m}$. The good direction shows sharp bits and track edges (Fig. 3, left). In the less favourable direction unsharp transitions and interrupted bits are visible (Fig. 3, right). The intrinsic magnetic structures beside the tracks have more or less the same dimensions as the written bits.

The extreme 1D behaviour of ME tape has allowed the development of simple quantitatively accurate models of the recording process. These models are based on extensions of the Williams and Comstock model [15] to thick media and to media with oblique easy axis angles [16,17] and are very similar, although they do not accurately predict the directional asymmetry of the output signal or the saturation of output with write current. These errors are associated with the limitations of the $1 \mathrm{D}$ model (in particular the neglect of the small but finite distribution of easy axis angles). Proper modelling of these details requires a more sophisticated approach.

2D self-consistent numerical models of the recording process in ME tapes have been reported [18-20]. The key simplifications in such models are the macroscopic approximations to the mode of reversal and the handling of interactions. As discussed in Ref. [3], most macroscopic models of the magnetic reversal process give rise to an inverse cosine dependence of the switching field on the angle between the field and the easy axis angle. Incoherent reversal can be incorporated in a Stoner-Wohlfarth model by appropriate truncation of the switching asteroid [19]. In the limit of very strong interactions mean field models are not appropriate. This does not seem to be the case for ME. A key feature that needs to be incorporated, however, is the cooperative interactions (which give rise to very sharp transitions) [19].

The results of the different numerical simulations are in good agreement and confirm the very $1 D$ nature of the recording process in these materials. Both the $\mathrm{ID}$ and more sophisticated models indicate that there is little or no improvement in the recorded output signal in ME tape associated with the transition from longitudinal to oblique recording $[16-19]$.

Recent noise measurements have provided further insight into the nature of the recording process in these

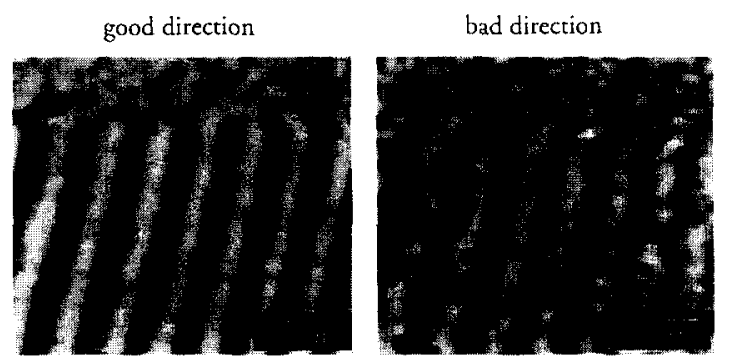

Fig. 3. MFM measurements of the recorded magnetization pattern in the good (left) and bad (right) recording directions. 
media [21]. The ac-erased tape noise is well described by a model which assumes that the noise is correlated through the depth of the tape. The ac-erased noise scales with the tape thickness, confirming that multilayer tapes have less noise than single-layer tapes [22]. The modulation noise was not a function of the carrier wavelength, suggesting that the noise in ME tape is stationary. These results are in agreement with previous results that indicate that there are no zigzag transitions in ME tapes [23]. The ac-erased and modulation noise results suggest (in agreement with Ref. [3]) that ME tape is more like a particulate medium than a thin-film medium (such as those used in rigid disk systems). The 'particles' in ME tape are associated with the obliquely oriented fibres or aggregates of fibres. This picture indicates that the oblique deposition process is more important in determining ME properties than the oblique recording process.

This macroscopic picture has its limitations. It is unable to explain the large variations in modulation noise which are observed as a function of recording direction [21,24]. A proper explanation of this result seems to require a micromagnetic approach. Preliminary micromagnetic results for the hysteresis properties of ME tape have been reported [25]. However, micromagnetic simulations suffer from a lack of knowledge of the variations in the medium as a function of depth (including composition and the easy axis angle), of the relationship between the effective easy axis angle associated with the mesoscopic columns and the crystalline anisotropy direction of the grains, of the distributions for the anisotropy coefficients and particle sizes, and of the spatial distribution of non-magnetic particles or voids. These questions represent the frontier in our attempts to understand the recording process in ME tape.

\section{Tribology and corrosion of ME tape}

In this section we discuss the tribological and corrosion properties of ME tape. The reliability of $\mathrm{ME}$ tape is of utmost importance because questions concerning the reliable operation of ME tape continue to hamper its use up to this day.

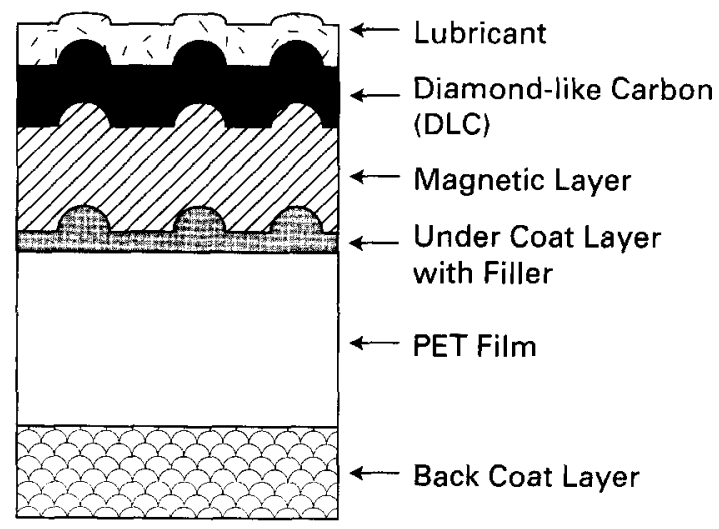

Fig. 4. Schematic cross section of an ME tape [26].
The tribological performance depends upon the details of the structure of the stack of layers used in ME tape. The basic structure of an advanced ME tape for digital video is shown in Fig. 4 [26]. From top to bottom, six layers can be distinguished. On top, there is a lubricant layer, often a fluorocarbon organic film about $4 \mathrm{~nm}$ thick. The next layer is a carbon film, in this case a diamond-like carbon (DLC) film (10 nm, [26]), which has two tasks: (a) it improves wear properties and (b) it separates the $\mathrm{Co}-\mathrm{O}$ film from the environment and improves corrosion resistance [26,27]. Large differences in the corrosion properties of different compositions of $\mathrm{Co}-\mathrm{O}$ film disappear when a DLC coating is applied [27]. This creates a great deal of freedom in the composition and structure of the metal (oxide) film that can be used to optimize recording output.

Below the DLC film the Co-O layer is found (see previous sections). On top of the PET substrate there is an undercoat with a filler which is very important for the performance of the ME tape because it provides a controlled roughness to the tape surface. This roughness prevents the tape sticking to the head and other parts of the recorder; this is an important factor for the wear rate of the heads and the occurrence of differential wear in the case of MIG heads [28]. This layer is one of the main improvements that made commercial introduction of ME tape possible. Below the substrate there is the back coat layer, which improves the runnability of the tape when it is in contact with the guidance parts of the mechanics in the recorder.

Investigations of wear of ME tape have been described extensively in the literature [28,29]. Tape degradation and failure is caused by the breaking away of parts of the metal coating, either by the high contact pressure or by fatigue of the metal film. The debris sticks to the head, accelerating tape wear, and a disaster occurs. It is clear that the adhesion of the metal film to the PET substrate has to be perfect and that the tape must be handled properly to avoid cracks.

To test corrosion the ME tape is subjected to harsh environmental conditions, as described in Refs. [27,30,31]. The ageing of ME tape is not fast compared with MP tape [30]. It appears that the substrate may play a part in the corrosion process [31] and the protection by the DLC layer is important [27].

Improvements in $\mathrm{ME}$ performance can be expected in the future. The metal coating can be improved because it can be separated from the (corrosive) environment. On the other hand, there will always be a need for protrusions to control the roughness and a need for a DLC-type coating for runnability. This may limit the improvements because a finite head-to-tape distance will be maintained.

\section{Conclusions and summary}

ME tape has matured; recording performance, corrosion and wear appear adequate, but our understanding of the 
variations of material properties, morphology and microstructure with preparation conditions is far from complete. The details of the oxidation process have to be studied in order to have a better control of the (magnetic) microstructure and to tailor the magnetic properties.

DLC-type coatings give enhanced protection from corrosion and allow a large degree of freedom for the composition of the magnetic thin film.

The recording process is properly described by macroscopic models. However, to understand the details of signal and noise micromagnetic models are required.

In most high-density helical scan systems equivalent ME and MP tapes are used. There is continuous competition between the two technologies. We expect that the trend in rigid disk systems to use thinner and thinner coatings to reach higher and higher densities will also occur in tape systems. This trend may favour ME tape in the long run.

\section{References}

[1] S.B. Luitjens. IEEE Trans. Magn. 24 (1990) 6.

[2] J. Hokkyo, T. Suzuki, K. Chiba, K. Sato, Y. Arisaka, T. Sasaki and Y. Ebine, J. Magn. Magn. Mater. 120 (1993) 281.

[3] H.J. Richter, IEEE Trans. Magn. 29 (1993) 2185.

[4] M. Futamoto and Y. Honda, J. Magn. Soc. Jpn. 18, S1 (1994) 485.

[5] T. Hinemo and T. Katahu, J. Magn. Magn. Mater. 134 (1994) 255.

[6] C. Yamamitsu, A. Iketani, J. Ohta and N. Echina, IEEE Trans. Magn. 31 (1995) 1037.

[7] H. Yoshida, K. Shinohara and M. Odagiri, J. Magn. Soc. Jpn. 18, S1 (1994) 439.

[8] G. Krijnen, S.B. Luitjens, R.W. de Bie and J.C. Lodder. IEEE Trans. Magn. 24 (1988) 1817.

[9] P. ten Berge, L. Abelmann, J.C. Lodder, A. Schrader and S.B. Luitjens. J. Magn. Soc. Jpn. 18, S1 (1994) 295.

[10] W. Meikeljohn and C.P. Bean, Phys. Rev. 105 (1957) 904.
[11] G. Bottoni, D. Candolfo and A. Cecchetti. IEEE Trans. Magn. 30 (1994) 3945.

[12] T. Takayama and K. Yoshida, J. Magn. Soc. Jpn. 13, S1 (1989) 819.

[13] J.P.C. Bernards and H.A.J. Cramer, IEEE Trans. Magn. 27 (1991) 4873.

[14] For example, N. Nouchi, H. Yoshida, K. Shinohara and A. Tomago, IEEE Trans. Magn. 22 (1986) 385.

[15] M.L. Williams and R.L. Comstock. AIP Conf. Proc. I (1971) 738.

[16] S.E. Stupp, S.R. Cumpson and B.K. Middleton. J. Magn. Soc. Jpn. 18, S1 (1994) 145.

[17] H.J. Richter, paper presented at SRS, Keele. UK (1994); H.J. Richter, IEEE Trans. Magn. 29 (1993) 2258.

[18] I. Tagawa, Y. Shimizu and Y. Nakamura, J. Magn. Soc. Jpn. 15, S2 (1991) 827.

[19] H.A.J. Cramer, PhD Thesis, University of Twente. The Netherlands, 1993.

[20] J.G. Zhu and D. Speliotis. J. Magn. Soc. Jpn. 18. S1 (1994) 111.

[21] S.E. Stupp and A.B. Schrader, IEEE Trans. Magn. 31 (1995) 2848.

[22] Y. Arisaka, K. Sato, Y. Yamada and K. Chiba. IEEE Trans. Magn. 27 (1991) 4742.

[23] K. Sato, K. Chiba, T. Ito, T. Sasaki and J. Hokkyo, J. Appl. Phys. 69 (1991) 4736.

[24] T. Ishida, R. Sugita and K. Tohma, 18th Collection of Summaries of Technical Papers of the Japan Appl. Magn. Soc. (1994) 14pG4.

[25] R.H. Victora, Phys. Rev. Lett. 58 (1987) 1788.

[26] H. Seki. H. Ueda, K. Kuwahara, M. Odagiri and T. Kunieda. J. Magn. Soc. Jpn. 18. S1 (1994) 291.

[27] K. Shinohara. H. Yoshida and T. Kunieda. J. Magn. Soc. Jpn. 18. SI (1994) 291

[28] T. Tsuchiya and B. Bhushan, IEEE Trans. Magn. 30 (1994) 4182.

[29] H. Osaki, K. Fukushi and K. Ozawa. IEEE Trans. Magn. 26 (1990) 3180.

[30] G. Fisher, H.J. Richter, H.P. Schildberg and H. Hibst. IEEE Trans. Magn. 29 (1990) 3757

[31] D.J. Peretti and D. Speliotis. J. Magn. Soc. Jpn. 18. S1 (1994) 279. 